\title{
Placement of p6pol between tandem repeat HIV-1 protease domains reduces Gag cleavage efficiency
}

\author{
Tin-An Chou', Kuo-Jung Huang ${ }^{2}$, Chin-Tien Wang ${ }^{1,2^{*}}$ \\ From Frontiers of Retrovirology: Complex retroviruses, retroelements and their hosts \\ Cambridge, UK. 16-18 September 2013
}

\section{Background}

HIV-1 protease (PR) is encoded by pol, which is initially translated as a $\operatorname{Pr} 160^{\text {gag-pol }}$ polyprotein by a ribosomal frameshift event [1]. Pr160 gag-pol is incorporated into virions via interactions with assembling $\operatorname{Pr} 55^{\text {gag }}$. The PRmediated proteolytic cleavage of $\operatorname{Pr} 55^{\text {gag }}$ and $\operatorname{Pr} 160^{\text {gag-pol, }}$, known as virus maturation, is essential for the acquisition of viral infectivity. Within the Gag-Pol, the p6gag is truncated and is replaced by a transframe domain referred to as $\mathrm{p}^{6 *}$ or p6pol. Removal of p6pol improves Gag-Pol autoprocessing, suggesting that p6pol is involved in regulation of PR activation [2]. However, overlapping of p6gag/p6pol reading frame hampers generic approach to studying p6pol biological function. To assess the p6pol contribution to PR-mediated virus maturation without affecting p6gag reading frame, we introduced an extra copy of p6pol-PR or PR coding sequence at the PR C-terminus.

\section{Materials and methods}

PCR-amplified p6pol-PR or PR fragments were inserted at the PR C-terminus of an env-deleted HIV-1 proviral vector. Each of the constructs was transiently expressed in $293 \mathrm{~T}$ cells, and virus assembly and processing were analyzed by Western blot. Virus infectivity was determined by a single-cycle infection assay.

\section{Results}

HIV-1 mutants containing tandem repeat PR domains were severely defective in virus particle production due to enhanced Gag cleavage. Inactivation of the proximal PR affects Gag cleavage efficiency at a greater extent than inactivation of the distal PR. Placement of p6pol between the tandem repeat $P R$ domains resulted in diminished Gag cleavage efficiency.

\section{Conclusions}

Our study indicates that the Gag cleavage enhancement effect incurred by over-expressed HIV-1 PR is reduced following the placement of p6pol between the tandem repeat PR domains. This supports the proposal that p6pol plays a negative role in the process of PR activation.

\section{Authors' details}

'Institute of Clinical Medicine, National Yang-Ming University School of Medicine, Taipei, Taiwan. ${ }^{2}$ Department of Medical Research and Education, Taipei Veterans General Hospital, Taipei, Taiwan.

Published: 19 September 2013

\section{References}

1. Jacks T, Power MD, Masiarz FR, Luciw PA, Barr PJ, Varmus HE: Characterization of ribosomal frameshifting in HIV-1 gag-pol expression. Nature 1988, 331:280-283.

2. Partin K, Zybarth G, Ehrlich L, DeCrombrugghe M, Wimmer E, Carter C: Deletion of sequences upstream of the proteinase improves the proteolytic processing of human immunodeficiency virus type 1. Proc Natl Acad Sci USA 1991, 88:4776-4780.

doi:10.1186/1742-4690-10-S1-P98

Cite this article as: Chou et al: Placement of p6pol between tandem repeat HIV-1 protease domains reduces Gag cleavage efficiency. Retrovirology 2013 10(Suppl 1):P98. 